\title{
Equilibrium states of a test particle coupled to finite size heat baths
}

\author{
Qun Wei, ${ }^{1}$ S. Taylor Smith, ${ }^{1}$ and Roberto Onofrio ${ }^{2,3,1}$ \\ ${ }^{1}$ Department of Physics and Astronomy,Dartmouth College, 6127 Wilder Laboratory, Hanover, NH 03755, USA \\ ${ }^{2}$ Dipartimento di Fisica "Galileo Galilei", Università di Padova, Via Marzolo 8,Padova 35131,Italy \\ ${ }^{3}$ Center for Statistical Mechanics and Complexity,INFM-CNR, Unità di Roma 1, Roma 00185, Italy
}

(Dated: April 8, 2022)

\begin{abstract}
We report on numerical simulations of the dynamics of a test particle coupled to competing Boltzmann heat baths of finite size. After discussing some features of the single bath case, we show that the presence of two heat baths further constrains the conditions necessary for the test particle to thermalize with the heat baths. We find that thermalization is a spectral property in which the oscillators of the bath with frequencies in the range of the test particle characteristic frequency determine its degree of thermalization. We also find an unexpected frequency shift of the test particle response with respect to the spectra of the two heat baths. Finally, we discuss implications of our results for the study of high-frequency nanomechanical resonators through cold damping cooling techniques, and for engineering reservoirs capable of mitigating the back-action on a mechanical system.
\end{abstract}

PACS numbers: 05.40.Jc, 05.70.Ln, 62.25.-g, 83.10.Rs

\section{INTRODUCTION}

Brownian motion is historically central to the molecular-level interpretation of statistical mechanics [1, 2], and it is crucial for precision measurements of macroscopic degrees of freedom [3]. More recently, Brownian motion has been interpreted as emerging from the interaction of a particle, classical or quantum, with an environment which may be schematized as a set of harmonic oscillators [4, 5], representing a heat bath. As the number of oscillators in the heat bath increases, the dynamics of the test particle considered as an open system becomes increasingly stochastic. Simple statistical laws for the average behavior of such open systems are obtained by considering environments with particular energy distributions, such as the canonical ensembles. Indeed, in the limit of an infinite number of oscillators, and for certain additional assumptions about their collective properties, including weak coupling and/or high temperature limits, the equation of motion for the test particle reduces to the familiar Langevin equation. In this case, stationary solutions correspond to the test particle's energy having a Boltzmann distribution in time, and therefore thermalized with the heat bath.

In many contexts, however, in particular for systems of interest in mesoscopic physics and nanotechnology, the size of the environment is small and does not justify this large number limit. There is also a natural infrared cut off for the frequency of the oscillators schematizing the environment, as the latter cannot support wavelengths much larger than its size. Furthermore, the finite amount of energy in any realistic enviroment demands an ultraviolet cut off in its density of states. Our study is aimed at understanding generic properties of thermalization in mesoscopic systems, and we add to the analytical results direct simulations of a test particle in contact with a finite number of oscillators, whose frequencies are distributed in a finite bandwidth. This is a textbook system, but it produces a range of behaviors which are missed by purely analytical studies, and which lead to a spectrum of phenomenological implications, in particular in the physics of high-frequency nanomechanical structures. Our findings may be relevant for the understanding of anomalies observed in the effective temperature of a resonating mode of a nanoresonator in interaction with a thermal bath and with the measurement apparatus schematized as a thermal bath at an effective temperature [6]. The nanoresonator dynamics result from the competition between two effective heat baths at different temperatures, in analogy to the phenomenon of cold damping first discussed in [3], and later demonstrated for macroscopic resonators in [7]. Anomalies observed in the experiment described in [6] may then be attributed to deviations from the Boltzmann distribution for the nanoresonator.

In a previous contribution [8], conditions under which thermalization occurs in the presence of a single finite resource heat bath have been discussed. In this paper, after recalling the case of thermalization in the presence of a single bath case and analyzing in more depth some specific issues, we continue our analysis by considering a situation in which the test particle is intermittently interacting with two different reservoirs. For Langevin reservoirs, this has been discussed, especially in recent years, in the literature [9 15. Langevin reservoirs, however, are only parameterized by the temperatures of the heat baths and the damping coefficients (or, alternatively, the relaxation times). In our case, the density of states of the heat baths, the number of particles in each bath, and the masses of the constituent particles are further parameters which influence the thermalization of the test particle. When two heat baths are present, they can also differ in temperature and density of states, opening up an even richer scenario. 
The paper is organized as follows. In Section II we discuss analytical results on heat baths, in the two extreme situations of a heat bath with infinite bandwidth and of zero bandwidth. Both cases can be treated analytically with simple approximations, and they both provide useful insights for the more general case of a finite bandwidth. In Section III we describe in some detail the numerical techniques and some results, complementing the ones presented in [8], about the dependence of thermalization upon the density of states and the approximate scaling of the thermalization curves. In Section IV we present the case of the two baths and examples of both thermalization and frustration of the test particle under intermittent interaction of the heat baths. The potential relevance of these studies to the analysis of recent experiments and possible future demonstrations is discussed in the conclusions, with particular regard to the possibility to engineer some baths to achieve approximated nondemolitive measurements in both the classical and quantum regime.

\section{SINGLE HEAT BATH: ANALYTICAL RESULTS}

Our starting point is the classic Hamiltonian of the test particle plus environment found in [4, 5, 16, 17]:

$$
H_{\mathrm{tot}}=\frac{P^{2}}{2 M}+\frac{1}{2} M \Omega^{2} Q^{2}+\sum_{n=1}^{N}\left[\frac{p_{n}^{2}}{2 m}+\frac{m \omega_{n}^{2}}{2}\left(q_{n}-Q\right)^{2}\right] .
$$

Here the test particle, with generalized coordinates $(Q, P)$, is schematized as a harmonic oscillator of mass $M$ and angular frequency $\Omega$, while the generic $n^{\text {th }}$ particle in the reservoir, with generalized coordinates $\left(q_{n}, p_{n}\right)$, is schematized as a harmonic oscillator of mass $m$ and angular frequency $\omega_{n}$. The systems are assumed to be onedimensional, and the coupling between the test particle and the particles in the reservoir has been chosen to be translationally invariant, to avoid the appearance of renormalization terms as discussed in [18, 19] (for more general couplings see also [20]).

To illustrate the impact of the bath frequencies $\left\{\omega_{n}\right\}$, we first consider two extreme situations for the range of their distribution which are analytically approachable: infinite bandwidth and zero bandwidth. The first situation is the usual Langevin approach, summarized here for completeness and to establish the notation we will use in the following. The case of a heat bath with zero bandwidth, i.e. all oscillators degenerate in frequency, is then studied, and we find that it produces a scaling law which can be approximated in the more realistic setting of a finite bandwidth.

\section{A. Heat bath with infinite bandwidth: Langevin scenario}

We briefly recall the major features of the Langevin approach and how it may be derived through the analysis of an ensemble of harmonic oscillators with the proper energy distribution. By writing the Hamilton equations from (1) and solving for the particles of the heat bath, the test particle can be described by the generalized Langevin equation [21, 22]:

$$
M \ddot{Q}(t)+\int_{t_{0}}^{t} d s \Gamma(t-s) \dot{Q}(s)+M \Omega^{2} Q(t)=\Pi(t)
$$

where the kernel of the dissipative term (in general non local in time) is:

$$
\Gamma(t-s)=\sum_{n} m \omega_{n}^{2} \cos \left[\omega_{n}(t-s)\right]
$$

and the fluctuation force term is:

$$
\Pi(t)=\sum_{n} m \omega_{n}^{2}\left\{\left(q_{n}\left(t_{0}\right)-Q\left(t_{0}\right)\right) \cos \left[\omega_{n}\left(t-t_{0}\right)\right]+\frac{p_{n}\left(t_{0}\right)}{m \omega_{n}} \sin \left[\omega_{n}\left(t-t_{0}\right)\right]\right\}
$$

In the limit of infinite harmonic oscillators in the bath extending over the continuum frequency range $\omega_{n} \in[0,+\infty)$, with density of states $d N / d \omega \propto 1 / \omega^{2}$, and if the initial conditions for the bath particles $\left(q_{n}\left(t_{0}\right), p_{n}\left(t_{0}\right)\right)$ are chosen 


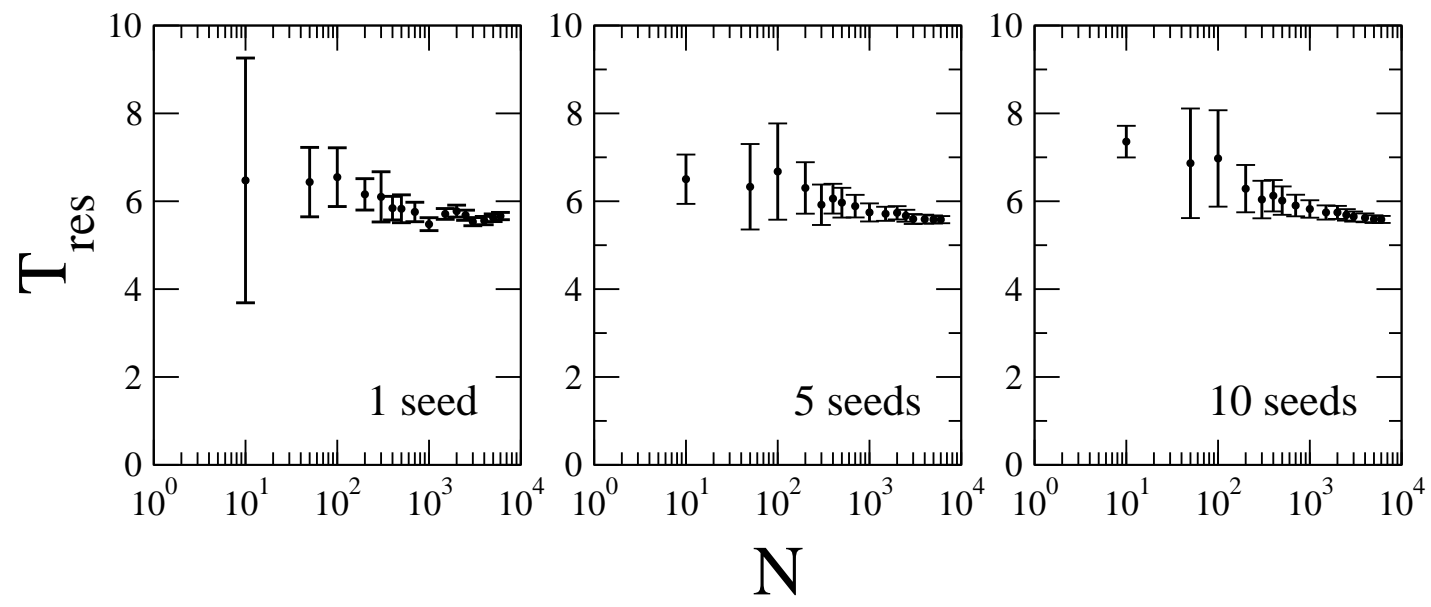

FIG. 1: Temperature of the heat bath versus the number of oscillators for three runs differing by the number of seeds. The bath temperature, determined by the fitting of each energy distribution with a Boltzmann curve, is the weighted average of the temperatures for each trial. The accuracy in the determination of the temperature increases significantly by increasing the number of oscillators and the number of seeds. In order to maintain reasonable times for the numerical experiments, we have chosen to work with 400 oscillators and 5-7 seeds in most of the single bath simulations.

to realize a Boltzmann energy distribution, the test particle will be described by a Langevin equation (for details see [23])

$$
d Q(t)=P(t) d t / M ; d P(t)=-\left[\gamma P(t)+M \Omega^{2} Q(t)\right] d t+\sqrt{2 m \gamma k_{B} T} d w(t)
$$

where $\gamma=\pi m \omega^{2} / 2 M \times d N / d \omega$. The stationary solution of Eqn. 5 corresponds to the thermalization of the test particle energy to the Boltzmann energy distribution of the bath.

\section{B. Degenerate heat bath: zero bandwidth}

In order to understand the importance of having the oscillators of the bath distributed in a large bandwidth we also discuss the extreme case of a test particle interacting with a finite number of oscillators which are degenerate in frequency $\left(\omega_{n}=\omega_{R}\right)$. In this case, if the initial conditions for the bath oscillators are distributed symmetrically in the phase space for each realization of the bath (such that $\sum_{n} q_{n}\left(t_{0}\right) \simeq 0, \sum_{n} p_{n}\left(t_{0}\right) \simeq 0$ ), the expressions for the dissipative kernel and the fluctuation force become, apart from stochastic corrections due to the partial cancellation in the initial conditions:

$$
\begin{gathered}
\Gamma(t-s)=N \omega_{R}^{2} \cos \left[\omega_{R}(t-s)\right] \\
\Pi(t)=-N m \omega_{R}^{2} Q\left(t_{0}\right) \cos \left[\omega_{R}\left(t-t_{0}\right)\right]
\end{gathered}
$$

The Langevin equation in this case is then written as:

$$
\ddot{Q}(t)-\xi \omega_{R}^{3} \int_{t_{0}}^{t} Q(s) \sin \left[\omega_{R}(t-s)\right] d s+(1+\xi) \omega_{R}^{2} Q(t)=0,
$$

where $\xi=N m / M$. The corresponding dynamics resembles that of multiple oscillators experiencing beating phenomena, with energy periodically transferred between the test particle and the heat bath as $E_{\mathrm{tp}}(t)=E_{0} \sin \omega_{R} t$. The corresponding energy probability density $P(E)$ for the test particle having energy between $E$ and $E+d E$ is therefore $P(E) \propto(d E / d t)^{-1} \propto\left[E\left(E_{0}-E\right)\right]^{-1 / 2}$. The absence of a bandwidth for the oscillators schematizing the heat bath is responsible for the lack of thermalization, as the test particle performs a deterministic, periodic motion, and in such a situation no thermalization can be expected. Nevertheless, this case is relevant for understanding the dependence of the thermalization upon the parameter $\xi$, as we will discuss in the next section. 


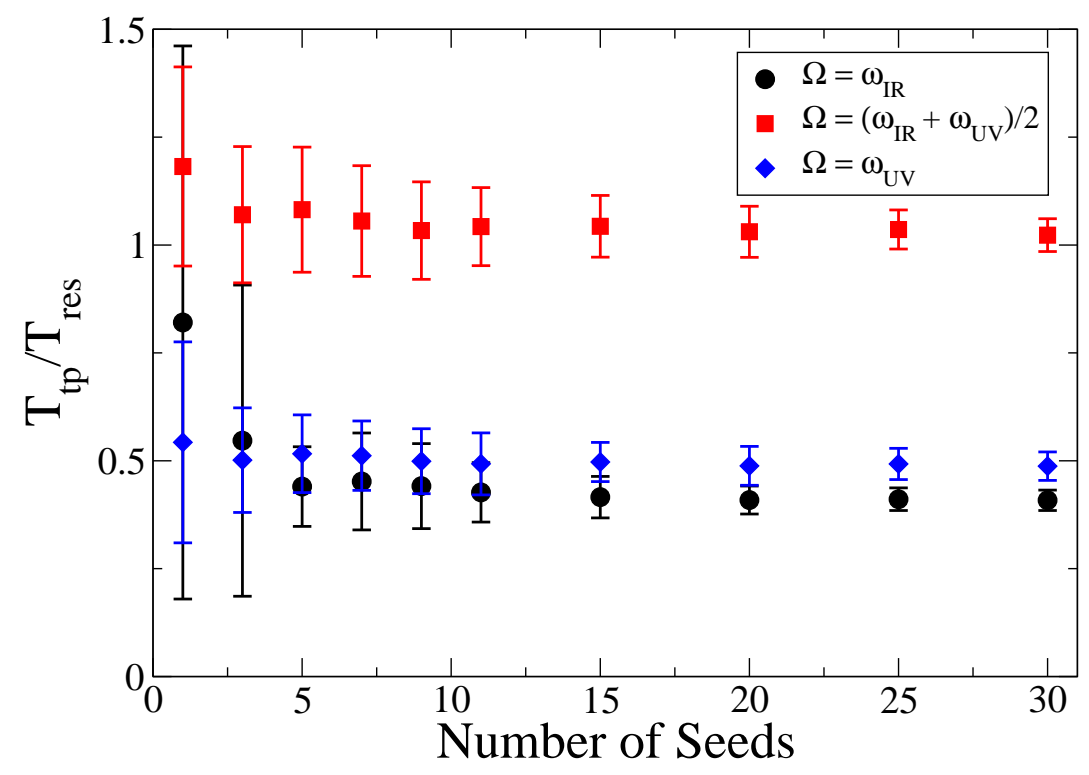

FIG. 2: (Color online) Temperature of the test particle in units of the temperature of the heat bath versus the number of trials, each one characterized by a different seed. The test particle is embedded in a bath made by 400 oscillators, and three situations are reported with different frequency of the test particle.

\section{SINGLE HEAT BATH: NUMERICAL RESULTS}

The above situations are highly idealized, since they both correspond to an infinite number of oscillators in the environment, either spread out over all possible frequencies or concentrated at a single frequency. As we have mentioned in the introduction there is a strong justification for relaxing these assumptions and considering the generic case of a finite environment with a finite bandwidth for the frequencies of its particles.

In a generic situation with the oscillators of the heat bath distributed in a finite bandwidth, no explicit solutions will generally be available. However, we can conjecture that the behaviour will lie in between the two extreme cases of the idealized Langevin heat bath and the degenerate heat bath. To corroborate this qualitative claim, we have used the method described below to numerically investigate the deviations from thermalization for a finite size reservoir characterized by a finite frequency spectrum $\omega_{n} \in\left[\omega_{\mathrm{IR}}, \omega_{\mathrm{UV}}\right]$, a finite number of oscillators, a finite mass ratio $m / M$ between the masses of the reservoir particles and the test particle, and a given density of states.

Strictly speaking, for a heat bath made of a finite number of oscillators coupled to a test particle, there will be no approach to equilibrium, as any initial state for the combined system will recur within a finite time [24 27]. If the number of oscillators in the bath is sufficiently large, however, the corresponding recurrence time will be very long. Provided one is interested in studying the dynamics on a timescale much shorter than this rather long recurrence time, an effective approach to equilibrium describable in terms of the usual thermodynamic quantities is viable. In this spirit, we focus our attention on the mutual relationship between the parameters of the heat bath and the test particle, to find the conditions under which the latter thermalizes with the former.

\section{A. Numerical approach to a finite size heat bath}

Our numerical approach consists of choosing values for the parameters which describe the test particle and the bath, solving the motion equations for the system, sampling the test particle's energy over time, and analyzing its distribution. The energies $\left\{E_{n}\right\}$ of the oscillators in the bath are chosen to follow a Boltzmann distribution, defining the bath temperature. The frequencies $\left\{\omega_{n}\right\}$ are chosen independently to follow a given-typically uniform-density of states, bounded by both the infrared and ultraviolet cutoffs. The $\left\{E_{n}\right\}$ and $\left\{\omega_{n}\right\}$ serve to constrain the initial conditions $\left\{\left(q_{n}\left(t_{0}\right), p_{n}\left(t_{0}\right)\right)\right\}$, which are then chosen to satisfy $E_{n}=\frac{p_{n}^{2}}{2 m}+\frac{1}{2} m \omega_{n}^{2} q_{n}^{2}$, each uniformly distributed in its available phase space. All of these values are chosen using the MATLAB pseudo-random number generator. In order to avoid bias among the frequencies and energies of the bath oscillators, we run each simulation multiple times, using different seeds for the generator, and average the results.

Having specified the quantities in the bath Hamiltonian, the original system of equations can be solved exactly - 


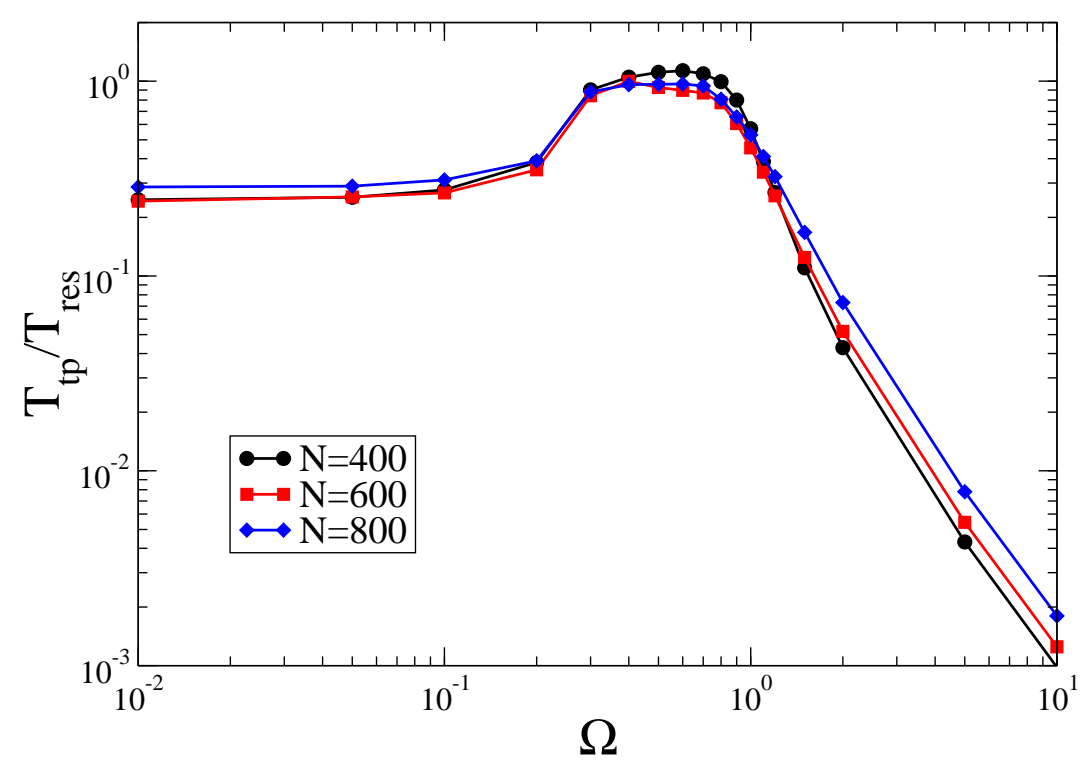

FIG. 3: (Color online) Thermalization plot with the temperature of the test particle in units of the temperature of the heat bath versus its frequency, for three different numbers of oscillators in the bath, $\mathrm{N}=400,600$, and 800 , and 15 seeds. The infrared cut-off frequency of the bath is $\omega_{\mathrm{IR}}=0.2$ and the ultraviolet cut-off frequency is $\omega_{\mathrm{UV}}=1$.

at least to the level of precision at which a matrix can be diagonalized numerically - using the following approach. The coordinates for each particle in the system are arranged into a vector $v$, with time derivative $\dot{v}$ :

$$
v(t)=\left(\begin{array}{c}
Q(t) \\
P(t) \\
q_{1}(t) \\
p_{1}(t) \\
\vdots \\
q_{N}(t) \\
p_{N}(t)
\end{array}\right), \quad \dot{v}(t)=\left(\begin{array}{c}
\dot{Q}(t) \\
\dot{P}(t) \\
\dot{q}_{1}(t) \\
\dot{p}_{1}(t) \\
\vdots \\
\dot{q}_{N}(t) \\
\dot{p}_{N}(t)
\end{array}\right)
$$

Using this notation, the system of $2 N+2$ equations describing the system-the Hamilton equations from Eq. 1 1 can be rewritten in matrix form as

$$
\dot{v}=A v
$$

where A is a square matrix of dimension $2 N+2$ given by

$$
A=\left(\begin{array}{ccccccccc}
0 & \frac{1}{M} & 0 & 0 & 0 & 0 & \cdots & 0 & 0 \\
-M \Omega^{2}-\sum_{n} m \omega_{n}^{2} & 0 & m \omega_{1}^{2} & 0 & m \omega_{2}^{2} & 0 & \cdots & m \omega_{N}^{2} & 0 \\
0 & 0 & 0 & \frac{1}{m} & 0 & 0 & \cdots & 0 & 0 \\
m \omega_{1}^{2} & 0 & -m \omega_{1}^{2} & 0 & 0 & 0 & \cdots & 0 & 0 \\
0 & 0 & 0 & 0 & 0 & \frac{1}{m} & \cdots & 0 & 0 \\
m \omega_{2}^{2} & 0 & 0 & 0 & -m \omega_{2}^{2} & 0 & \cdots & 0 & 0 \\
\vdots & \vdots & \vdots & \vdots & \vdots & \vdots & \ddots & \vdots & \vdots \\
0 & 0 & 0 & 0 & 0 & 0 & \cdots & 0 & \frac{1}{m} \\
m \omega_{N}^{2} & 0 & 0 & 0 & 0 & 0 & \cdots & -m \omega_{N}^{2} & 0
\end{array}\right) .
$$

This matrix can be written as $A=C D C^{-1}$, where $D$ is the diagonal matrix of eigenvalues, and $C$ is the matrix of corresponding eigenvectors. We can then rewrite the matrix equation (9) as $\dot{v}=C D C^{-1} v$ and then $C^{-1} \dot{v}=D C^{-1} v$. If we let $v^{\prime}=C^{-1} v$ and $\dot{v}^{\prime}=C^{-1} \dot{v}$ then we have a new matrix equation in normal coordinates $\dot{v}^{\prime}=D v^{\prime}$. This yields a set of independent, second order, homogenous differential equations $\dot{v}_{n}^{\prime}=d_{n} v_{n}^{\prime}$ where the $\left\{d_{n}\right\}$ are the diagonal elements of $D$, the eigenvalues of $A$. Each of these equations has the simple solution $v_{n}^{\prime}=v_{0_{n}}^{\prime} e^{d_{n} t}$ where $v_{0_{n}}^{\prime}$ is the $n^{\text {th }}$ 


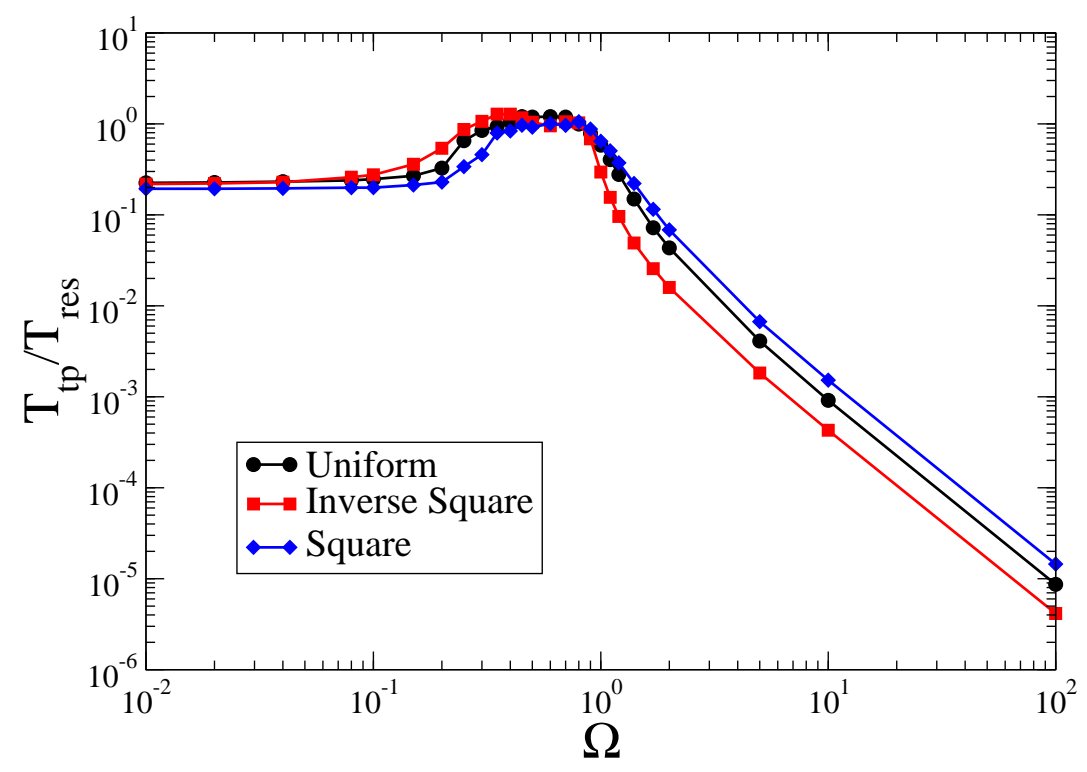

FIG. 4: (Color online) Thermalization curves for a test particle interacting with heat baths having frequencies in the range between 0.2 and 1, distributed uniformly, and with inverse square and square dependence on the frequency in the same bandwidth. The presence of more oscillators at lower or higher frequencies induces thermalization at lower or higher frequencies of the test particle, respectively.

component of the vector of initial conditions in normal coordinates, obtained by applying $C^{-1}$ to the initial conditions $v_{0}$. We can write these solutions in a vector

$$
v^{\prime}(t)=\left(\begin{array}{c}
v_{0_{1}}^{\prime} e^{d_{1} t} \\
v_{0_{2}}^{\prime} e^{d_{2} t} \\
\vdots \\
v_{0_{N}}^{\prime} e^{d_{N} t}
\end{array}\right)
$$

The coordinate vector containing the position and momentum of the test particle and bath particles at any time $t$ can then be obtained by substituting the value of $t$ into $v^{\prime}(t)$ and multiplying by the matrix $C$ to return to the original coordinates.

Since $C$ has the same dimensions as $A$ - specifically, $(2 N+2) \times(2 N+2)$-this causes the process of determining the state of the system at some time to run in time which is $O\left(N^{2}\right)$ in the number of particles in the bath. However, it is possible to avoid this issue of computational resources if we are willing to sacrifice knowledge about the state of each bath particle at time $t$, and simply request the knowledge of the position and momentum $q$ and $p$ of the test particle. In this case we can simply multiply the vector $v^{\prime}(t)$ by the first row of $C$ to obtain the value of $q$, and by the second row of $C$ to obtain the value of $p$-then the process runs instead in $O(N)$, which significantly speeds up the simulations for relatively large numbers of oscillators.

The thermal behavior of the test particle can be observed by sampling its position and momentum at different times and calculating its energy, and then making a histogram of the measured energies. If the distribution of energies follows the Boltzmann law, we say the test particle has thermalized, and we can define a temperature for the test particle by looking at the slope of the distribution. This temperature can then be compared with the temperature of the original energy distribution of the bath particles. The slope of the distribution is evaluated in a separate function by taking the natural logarithm of the height of each bin, and then performing weighted least squares fitting, with the weight of each data point being given by $N_{i}$, the original height of the bin. The statistical fluctuations in the energy are expected to have a Poisson distribution, which if centered around a value $x$ has a standard deviation of $\sigma=\sqrt{x}$, and thus the uncertainty in the number of elements $N_{i}$ in each bin is given by $\sigma_{i}=\sqrt{N_{i}}$. Since the weights in a weighted least squares fit are given by $w_{i}=\frac{1}{\sigma_{i}^{2}}$, the weights for fitting $\ln N$ are then $N_{i}$. The relatively large number of oscillators and the statistical accuracy of the results do not require fitting with a canonical power law distribution as discussed in [28, 29, 31 33].

One issue in sampling the energy of the test particle is considering the time scales involved in the problem, and possible periodicity. Any sampling corresponding to periodicities in the motion of the test particle may produce 


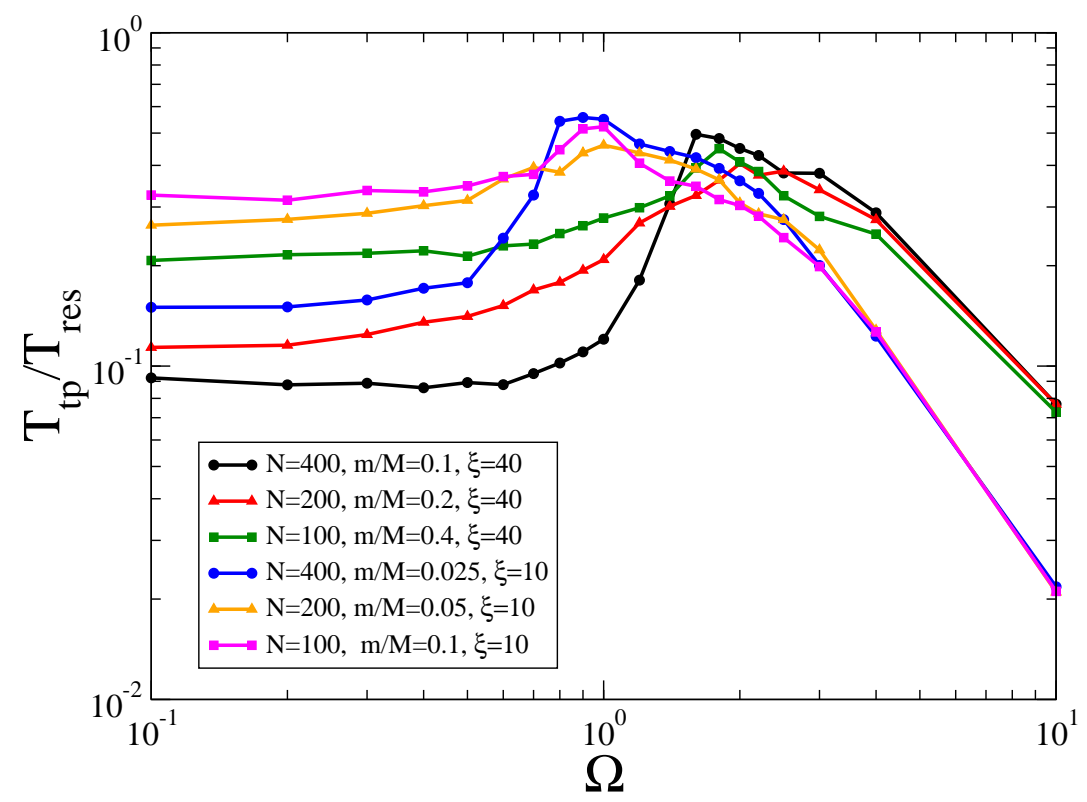

FIG. 5: (Color online) Approximated scaling properties of the thermalization peak upon the parameter $\xi=N m / M$. Shown is the ratio between the temperature of the test particle and the heat bath temperature versus the proper frequency of the test particle near the optimal thermalization region for two different values of $\xi$. The three curves within each value of $\xi$ differs by the number of particles and the mass ratio, respectively $N=100,200,400$ and $m / M=0.1,0.05,0.025$ for the case of $\xi=10$, and $N=100,200,400$ and $m / M=0.4,0.2,0.1$ for the case of $\xi=40$. Notice that for the same $\xi$ value the thermalization peaks occur at approximately the same frequency. The case of a degenerate bath would predict a ratio between the frequencies at which the peaks of thermalization occur as $\sqrt{41 / 11} \simeq 2$ in approximate agreement with the observed pattern.

misleading results. For this reason we use random step sizes in sampling the test particle energy, with the distribution of random step sizes centered around a chosen value, uniformly distributed between zero and twice that value, in such a way that the latter represents the average sampling time.

The precision in the determination of the temperature of the heat bath and the test particle are due to different factors. The precision of the bath temperature is limited by the number of oscillators it comprises, which is generally in the hundreds. Even generating multiple realizations of the bath energies and averaging over these to compensate for the quality of the distribution produced by the random number generator does not result in significant improvements here. This is shown in Fig. 1 where the heat bath temperature and its standard deviation are plotted versus the number of oscillators for three different numbers of realizations, each of which represents a different seed for the random number generator. For the test particle temperature, on the other hand, the precision at leading order is determined by the chosen number of random realizations. Averaging over a large number of trials lowers the influences from bad seeds when generating the energy and frequency distributions of the bath particles, as can be seen in Fig. 2. However, too many trials will result in a very long simulation time. For those tests which require high precision we in general use 15 seeds, while for tests requiring less precision 5-7 seeds are used.

\section{CONDITIONS FOR THERMALIZATION OF THE TEST PARTICLE TO A HEAT BATH}

Examples of partial and complete thermalization of the test particle to the reservoir are shown in Fig. 3 for different numbers of oscillators in the heat bath (see [8] for examples of the test particle energy distribution). We find three regimes: at low angular frequencies $\Omega<<\omega_{\text {IR }}$, the test particle approaches an equilibrium state corresponding to an average energy lower than the reservoir temperature, its spectral dependence is flat, and the energy distribution is significantly different from the Boltzmann one, as discussed in [8]. In the intermediate regime $\omega_{\mathrm{IR}} \leq \Omega \leq \omega_{\mathrm{UV}}$, the test particle generally approaches complete thermalization. In the region where $\Omega>>\omega_{\mathrm{UV}}$, the test particle thermalizes at a temperature significantly lower than the one of the reservoir, with a strong dependence upon the frequency. We have tested the dependence of the thermalization plot on the choice of the density of states of the heat bath, as show in Fig. 4. Keeping all the remaining parameters constant, the density of states is chosen to be proportional to $1 / \omega^{2}$, independent of $\omega$, or proportional to $\omega^{2}$ in the three plots, in all cases within the same spectral interval (and zero otherwise). It is apparent that for the global thermalization features it makes little difference 

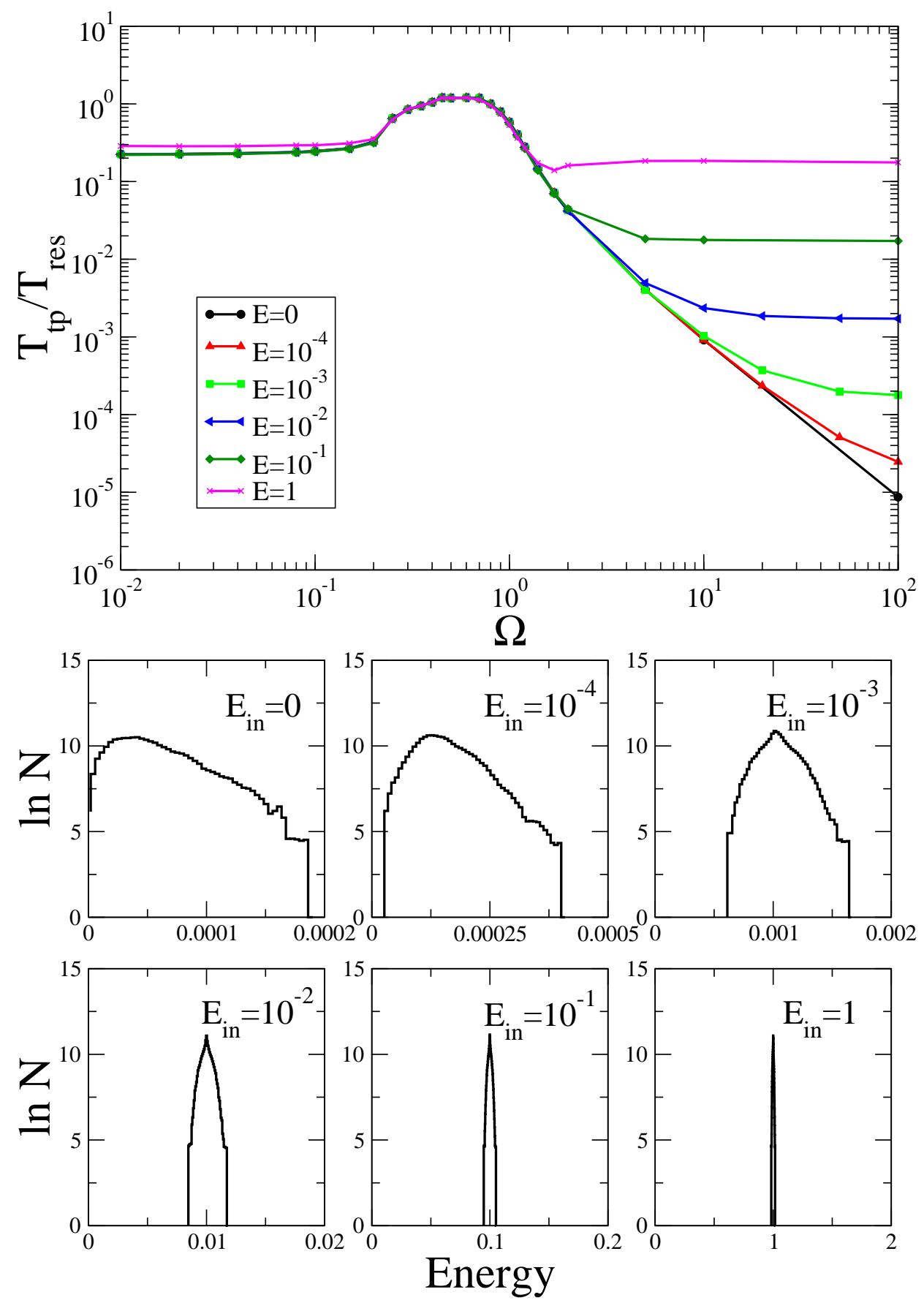

FIG. 6: (Color online) Top plot: thermalization curves for a test particle interacting with heat baths having frequencies in the range between 0.2 and 1, distributed uniformly, for different values of the test particle initial energy. At high frequency the test particle is effectively decoupled from the heat bath and therefore maintains its initial energy. Bottom plot: energy distribution for the test particle with $\Omega=100$ versus its initial energy, with the bath temperature being $T=5.5$ in arbitrary units. The distribution appears Boltzmann at low energies, but becomes Gaussian at high energies, with its peak around the initial energy.

whether the frequencies follow a uniform, square, or inverse square distribution; the only difference is visible at the edges of the peak thermalization region, where the presence of more oscillators at lower or higher frequencies induces thermalization at slightly lower or higher frequencies of the test particle, respectively. Considering the similarity among the various choices for the density of states, we have used the simplest uniform distributions in all subsequent simulations.

The thermalization region can be understood by thinking of the case of a degenerate heat bath: if the test particle 

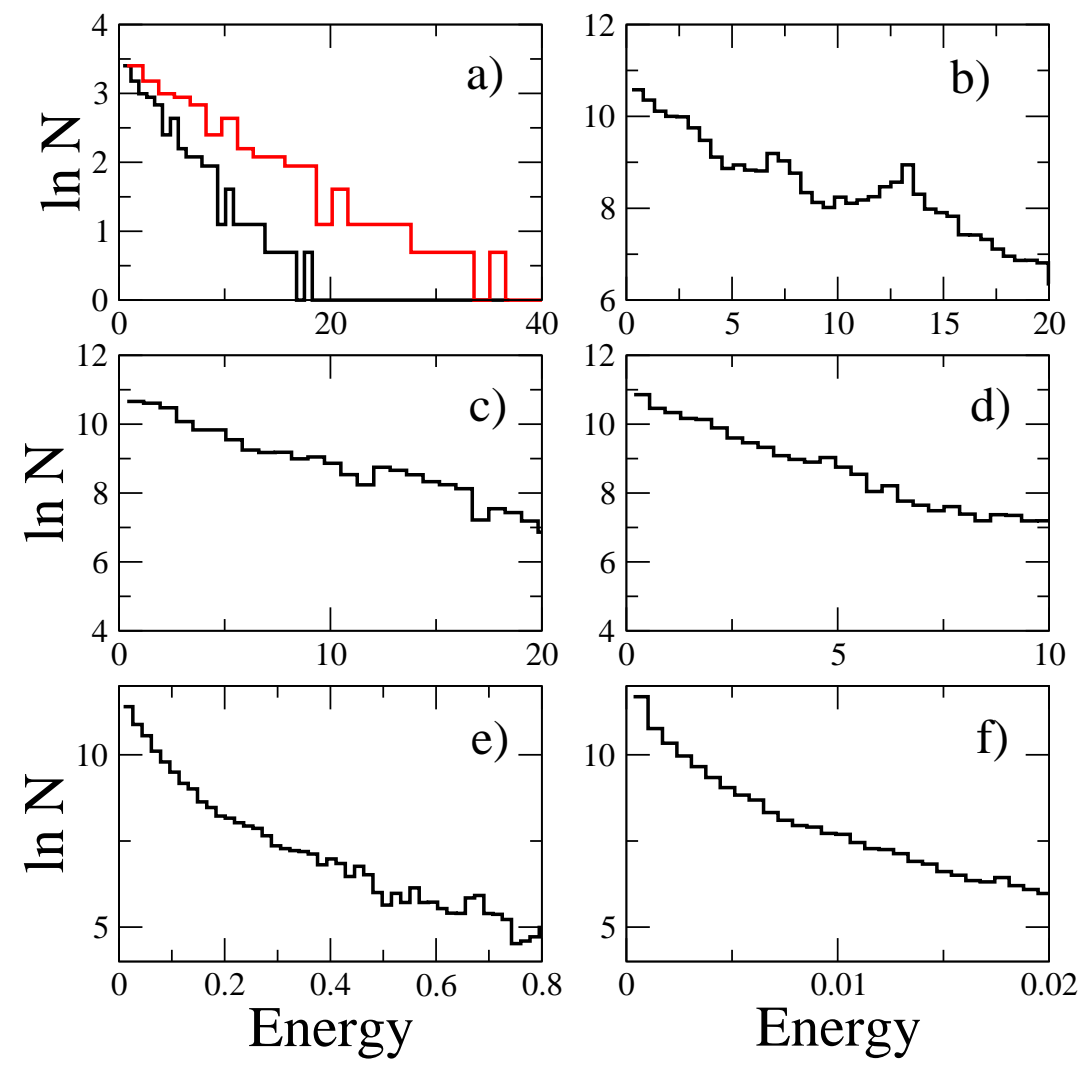

FIG. 7: (Color online) Energy distribution in the case of a test particle intermittently interacting with two baths at different temperature. In the top left plot the energy distribution for the two baths at temperatures 5 and 10 respectively is depicted, while in the other plots the energy distribution for the test particle for different values of its frequency is shown (from $b$ to $f$, $\Omega=0.01,0.3,0.6,2,10)$. In all the simulations we have used 200 oscillators in each heat bath, with a mass ratio between the bath particle and the test particle $m / M=10^{-3}$, and a switching time $\Delta T=1$ in units of time steps.

is in resonance with the bath oscillators, it will trade energy with them in an efficient way, and otherwise the energy exchange will be limited both in amplitude and speed. That the test particle thermalizes generally near the bandwidth of the heat bath is thus to be expected, although in some cases the effective frequency of the test particle in contact with the bath is sufficiently different than its natural frequency $\Omega$ that the region of peak thermalization is shifted. Since the effective frequency under the effect of the heat bath is renormalized as $\tilde{\Omega}=(1+\xi)^{1 / 2} \Omega$, if $\xi$ is significantly larger than zero we expect the resonance to occur at angular frequencies higher than the intrinsic angular frequency of the test particle, and this should only depend on $\xi$. The shifts of the thermalization regions seen in Fig. 5 are in agreement with this scaling hypothesis. This has as a further implication a non monotonic dependence of the thermalization upon the mass ratio $m / M$, which cannot be trivially addressed in a perturbative approach 34]. As already discussed in [8], the thermalization efficiency of the test particle at a given oscillation frequency is initially directly proportional to the mass ratio $m / M$ determining the coupling strength of the bath-test particle interaction, and then reaches an optimal value before the $\xi$-dependent renormalized frequency plays a role.

Another phenomenon worthy of note occurs in the high frequency region, where the thermalization curve is dependent upon the initial conditions of the test particle, as it is evident from the thermalization plots in the top plot of Fig. 6. While at lower frequencies the particle thermalizes to the same temperature regardless of its initial energy, at higher frequencies it is effectively decoupled from the lower frequency heat bath. These energy distributions are reported in the bottom plots of Fig. 6. This non-Markovian behavior can be understood as the classical counterpart of the dynamical decoupling techniques developed to protect a quantum system from environmentally induced decoherence [35], in which a system driven at a frequency much higher than the ultraviolet cut off of the bath is effectively decoupled. In this case the test particle oscillation frequency naturally provides a dynamical self-decoupling mechanism. 

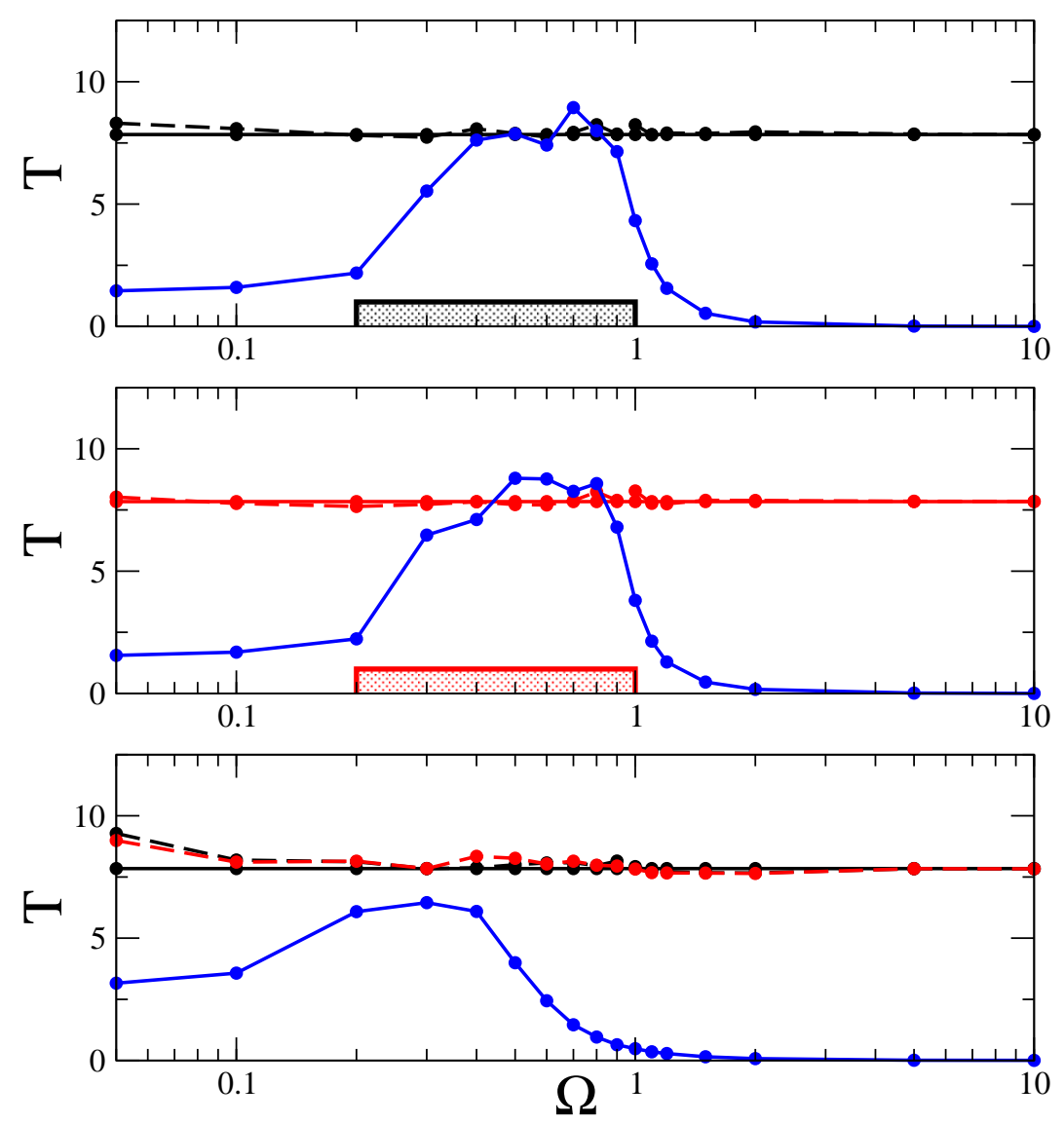

FIG. 8: (Color online) Thermalization plots for the case of two heat baths in interaction with a test particle versus its proper frequency. In the top plot the case of the test particle only interacting with a heat bath at temperature 7.5 (in arbitrary units) is reported (blue curve), together with the initial and final temperature of the heat bath acting on it (black curves, continuous and dashed, respectively). In the middle plot the test particle is interacting with another heat bath at the same initial temperature of 7.5 and the same density of state as the lower temperature bath. In the bottom plot the effect of the two baths is shown when they intermittently interact with the test particle. Notice that optimal thermalization occurs at frequencies lower than in the case of each bath acting independently, leading to an effective temperature lower than the common one of the two heat baths.

\section{DYNAMICS IN THE PRESENCE OF TWO THERMOSTATS}

Here we discuss the case of a test particle connected to two heat baths with finite resources. In order to avoid simultaneous contact between the two baths through the test particle, which will eventually lead to their complete thermalization, we consider an intermittent interaction between the test particle and the baths. In this way, as also tested at the end of each simulation, the two baths still preserve their original temperature and energy distribution for a weak bath-test particle coupling.

The total Hamiltonian for the system made of the two baths and the test particle is given by

$$
H_{\text {tot }}=\frac{P^{2}}{2 M}+\frac{1}{2} M \Omega^{2} Q^{2}+\sum_{n=1}^{N 1}\left[\frac{p_{1_{n}}^{2}}{2 m}+\frac{1}{2} m \omega_{1_{n}}^{2}\left[q_{1_{n}}-\alpha_{1}(t) Q\right]^{2}\right]+\sum_{n=1}^{N 2}\left[\frac{p_{2_{n}}^{2}}{2 m}+\frac{1}{2} m \omega_{2_{n}}^{2}\left[q_{2_{n}}-\alpha \alpha_{2}(t) Q\right]^{2}\right] .
$$

where $\alpha_{1}(t)=1$ if $2 k \Delta T<t<(2 k+1) \Delta T$ and $\alpha_{1}(t)=0$ if $(2 k+1) \Delta T<t<(2 k+2) \Delta T$, and $\alpha_{2}(t)=1-\alpha_{1}(t)$ (with $k$ an integer number), and $\Delta T$ is the time the test particle is in contact with each bath. By expanding the $\left(q_{n}-Q\right)^{2}$ terms, as in the one bath case, the Hamiltonian can be rewritten as 

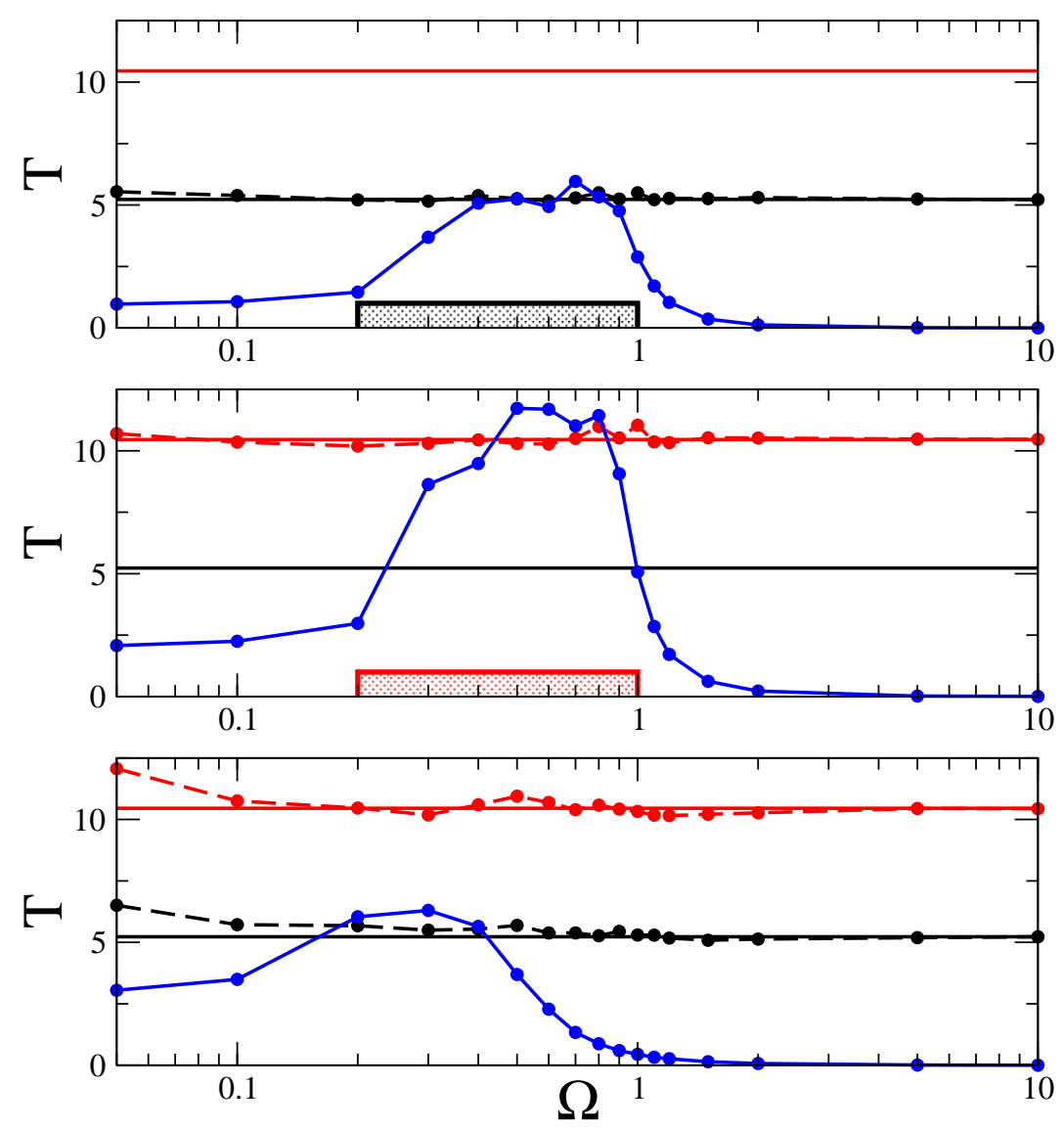

FIG. 9: (Color online) Thermalization plots for two heat baths in interaction with a test particle versus its proper frequency. In the top plot the case of the test particle only interacting with a heat bath at temperature 5 (in arbitrary units) is reported (blue curve), together with the initial and final temperature of the heat bath acting on it (black curves, continuous and dashed, respectively). In the middle plot the test particle is instead interacting with a heat bath at the initial temperature of 10 , having the same density of state as the lower temperature bath. In the bottom plot the effect of the two baths is shown when they intermittently interact with the test particle. Notice that optimal thermalization occurs at frequencies lower than in the case of each bath acting independently.

$$
\begin{aligned}
H_{\mathrm{tot}} & =\frac{P^{2}}{2 M}+\frac{1}{2} M\left[\Omega^{2}+\alpha_{1}(t)^{2} \sum_{n=1}^{N 1} \frac{m}{M} \omega_{1_{n}}^{2}+\alpha_{2}(t)^{2} \sum_{n=1}^{N 2} \frac{m}{M} \omega_{2_{n}}^{2}\right] Q^{2} \\
& +\sum_{n=1}^{N 1}\left[\frac{p_{1_{n}}^{2}}{2 m}+\frac{1}{2} m \omega_{1_{n}}^{2} q_{1_{n}}^{2}\right]+\sum_{n=1}^{N 2}\left[\frac{p_{2_{n}}^{2}}{2 m}+\frac{1}{2} m \omega_{2_{n}}^{2} q_{2_{n}}^{2}\right] \\
& -\left[\sum_{n=1}^{N 1} \alpha_{1}(t) m \omega_{1_{n}}^{2} q_{1_{n}}+\sum_{n=1}^{N 2} \alpha_{2}(t) m \omega_{2_{n}}^{2} q_{2_{n}}\right] Q
\end{aligned}
$$

The second, third, and fourth terms in (12) correspond to the first bath independent of the test particle, the second bath independent of the test particle, and the interaction of the two baths with the test particle, respectively.

The equations of motion can be derived from the Hamiltonian, and the MATLAB code for the case of two baths operates in the same way as the one bath case. The $\left\{q_{2_{n}}\right\}$ and $\left\{p_{2_{n}}\right\}$ for the second bath are simply appended to the vector of coordinates for the system $v$, so that $v$ and $\dot{v}$ have the form: 


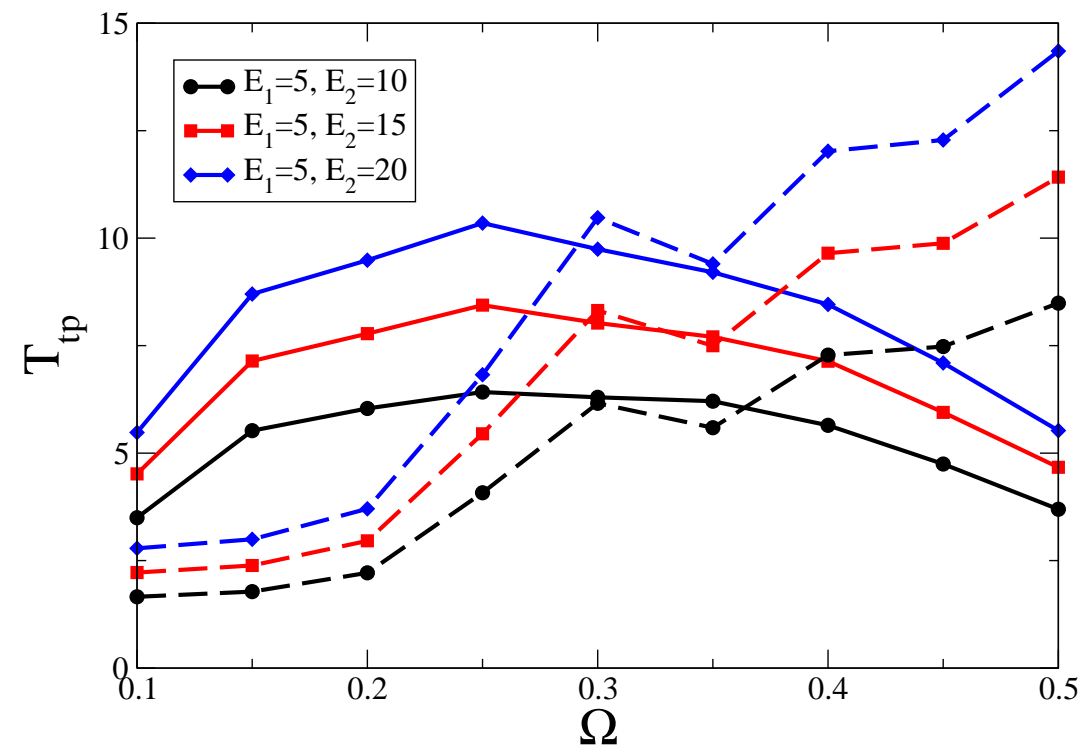

FIG. 10: (Color online) Thermalization plots for two heat baths in interaction with a test particle versus its frequency (solid lines), and comparison with the arithmetic average of the two temperatures when the test particle interacts with the two baths separately (dashed lines). The colder bath is kept at temperature 5 (in arbitrary units), and the other bath is at temperature 10, 15 and 20 respectively for black, red and blue lines, with a uniform density of states for the oscillators of the two baths between $0.2 \Omega$ and $\Omega$, and zero elsewhere. Notice that the resulting effective temperature of the test particle is close to the arithmetic average of the temperatures of the two baths only in a narrow region around $0.3-0.35 \Omega$.

$$
v(t)=\left(\begin{array}{c}
Q(t) \\
P(t) \\
q_{1_{1}}(t) \\
p_{1_{1}}(t) \\
\vdots \\
q_{1_{N_{1}}}(t) \\
p_{1_{N_{1}}}(t) \\
q_{2_{1}}(t) \\
p_{2_{1}}(t) \\
\vdots \\
q_{2_{N_{2}}}(t) \\
p_{2_{N_{2}}}(t)
\end{array}\right), \quad \dot{v}(t)=\left(\begin{array}{c}
\dot{Q}(t) \\
\dot{P}(t) \\
\dot{q}_{1_{1}}(t) \\
\dot{p}_{1_{1}}(t) \\
\vdots \\
\dot{q}_{1_{N_{1}}}(t) \\
\dot{p}_{1_{N_{1}}}(t) \\
\dot{q}_{2_{1}}(t) \\
\dot{p}_{2_{1}}(t) \\
\vdots \\
\dot{q}_{2_{N_{2}}}(t) \\
\dot{p}_{2_{N_{2}}}(t)
\end{array}\right)
$$

The system of Hamilton equations describing the motion of the system, now of size $2\left(N_{1}+N_{2}\right)+2$, can be replaced by the matrix equation $\dot{v}=A v$, where $A$ has the similar form as (10), but now has dimension $\left[2\left(N_{1}+N_{2}\right)+2\right] \times$ $\left[2\left(N_{1}+N_{2}\right)+2\right]$. Since in our test the two baths are not simultaneously in contact the test particle, two matrices are constructed: $A_{1}$ in which all the terms of bath 2 are set to zero and $A_{2}$ in which all terms of bath 1 are instead set to zero. The parameters for each of the two baths are allowed to be specified independently. In principle, this also allows for an arbitrary partition of $N$ harmonic oscillators in $\ell$ different heat baths, each specified by different initial conditions and density of states. The matrix diagonalization method used in one-bath thermalization to solve the equations will not work now since it assumes the matrix $A$ does not change over time. In the two-bath case instead $A$ switches between $A_{1}$ and $A_{2}$, simulating the test particle switching between the two baths. Therefore we use a fourth order Runge-Kutta method to solve the equations, switching between $A_{1}$ and $A_{2}$ at each step ( $\Delta T=1$ in units of time steps of the Runge-Kutta code). This fast switching is the closest scenario to the case of a continuous interaction of the test particle with the two baths, maximizing the frustration of the test particle in equilibrating to each. Simulations with switching times $\Delta T$ of 2,10 , and 20 time steps have been also performed yielding indistinguishable energy distributions within the statistical error. In the case of slow switching, i.e. for $\Delta T$ much longer than the thermalization time, the dynamics is complicated by the fact that the test particle, once thermalized with one bath, will have an initial condition with finite energy which can create a situation similar to the one discussed around Fig. 6 . 

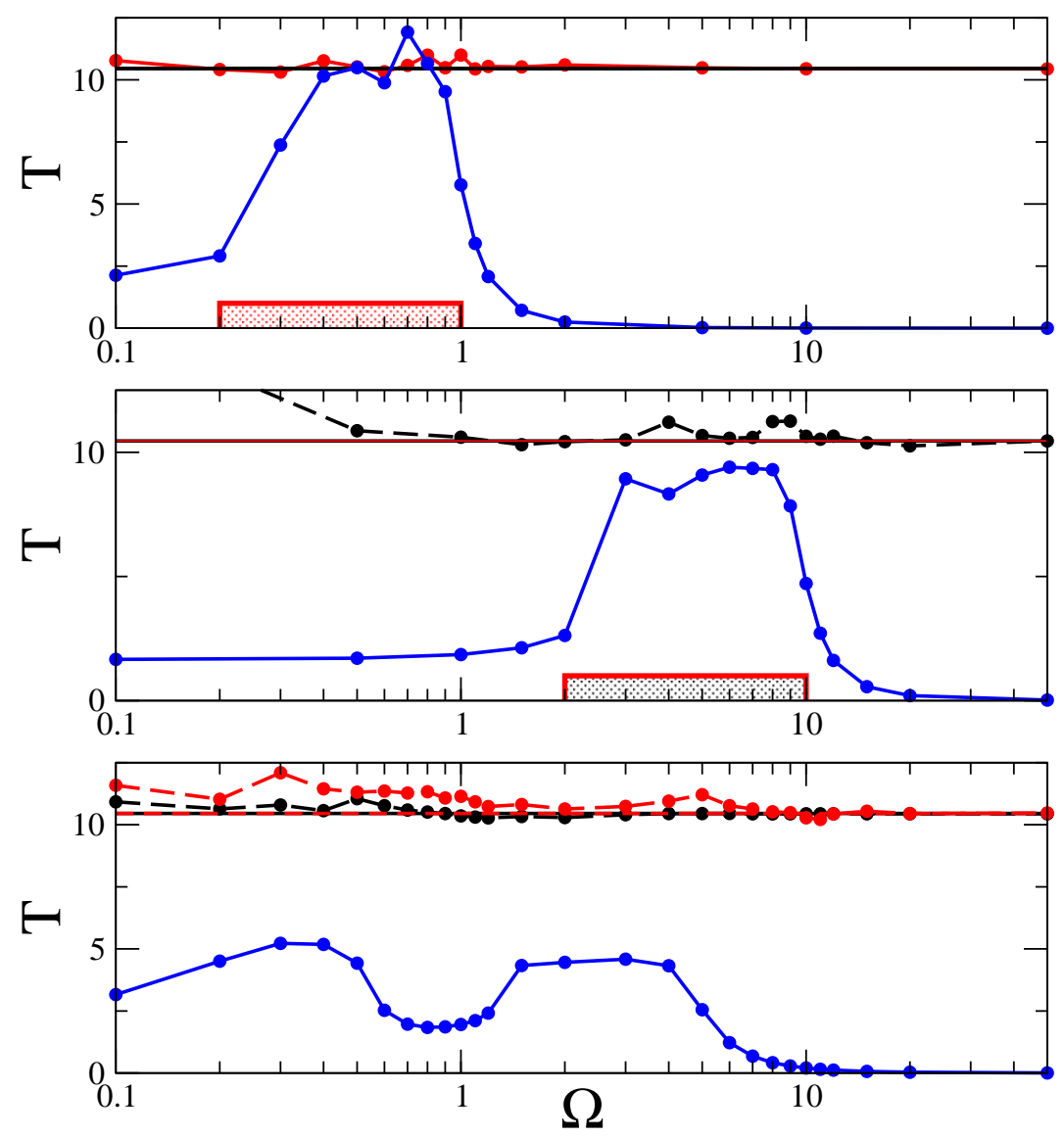

FIG. 11: (Color online) Thermalization plots for two heat baths in interaction with a test particle versus its proper frequency. The two heat baths have the same temperature 10 (in arbitrary units) and different, non-overlapping, density of states (see top and center figures). When only one bath is acting, the test particles thermalizes to the corresponding bath if its frequency is in the same spectral region as the density of states of the bath. In the bottom plot the effect of the two baths is shown when they intermittently interact with the test particle, with two different regions of partial thermalization.

Therefore thermalization may be actually affected by the energy acquired by the test particle prior to the interaction with the next bath. In the simplest case of two infinite bandwidth Langevin baths as the one discussed in Section II.A, a thermalization to one bath first, and then to the other bath, is expected instead, regardless of the test particle energy. In this case the energy distribution for the test particle in the long time limit is expected to be the sum of two Boltzmann distributions, i.e.

$$
P(E)=\frac{1}{2}\left[\beta_{1}^{-1} \exp \left(-\beta_{1} E\right)+\beta_{2}^{-1} \exp \left(-\beta_{2} E\right)\right]
$$

with $\beta_{i}=\left(K_{B} T_{i}\right)^{-1}, i=1,2$ the inverse temperature. We have also verified using the one-bath case that for a sufficiently small step size the Runge-Kutta method reproduces the results obtained using numerical diagonalization.

Fig. 7 shows the energy distribution in the case of the test particle interacting with two baths at different temperatures. Similar to the one bath case shown in [8], the energy distribution of the test particle reaches a Boltzmann distribution when the frequency of the test particle is within the finite frequency spectrum of the bath particles, while the distribution becomes progressively less Boltzmann when the frequency of the test particle moves outside the spectrum. Fig. 8 shows the effect of the two baths with nominal equal temperature and density of states. The usual single bath thermalization curves when each of the baths is acting on the test particle are depicted in the top and middle plots, while the thermalization plot corresponding to their intermittent action is shown in the bottom plot. It is worth noticing that the optimal thermalization region of the test particle is shifted towards lower angular frequencies, and its temperature is slightly lower than the common temperature of the baths. In close analogy to the one bath case, one can then change the temperature of the two heat baths while keeping their density of state equal to each other. As shown in Fig. 9, it is confirmed that the test particle's thermalization region is shifted towards significantly lower frequencies with respect to the thermalization curves for each separate heat bath. Also, 

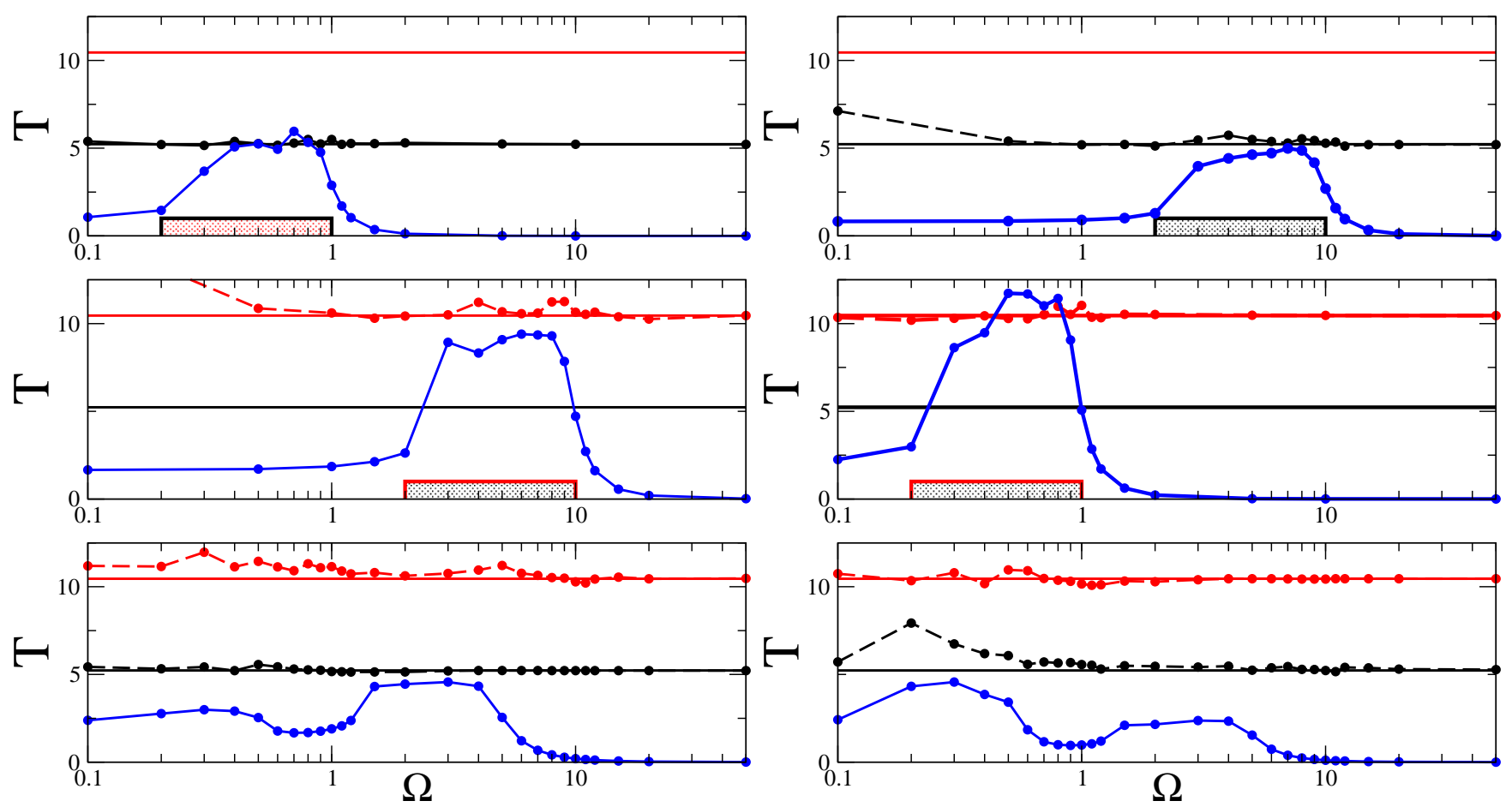

FIG. 12: (Color online) Thermalization plots for two heat baths in interaction with a test particle versus its proper frequency. The two heat baths have both different temperature and density of states, as visible in the top and center plots, with the colder bath (at $\mathrm{T}=5$ in arbitrary units) having the bandwidth centered at lower frequency range (0.2 to 1$)$ in the left plot, and centered at higher frequency range (2 to 10) in the right plot. In the bottom plot the effect of the two baths is shown when they intermittently interact with the test particle, which is frustrated both by the different temperatures and the different density of states.

the test particle is observed to thermalize at a temperature lower than the average of the temperatures of the two baths. The average of the temperatures of the two baths is the effective temperature of the test particle expected for $E<<K_{B} T_{1}, K_{B} T_{2}$ in the adiabatic switching limit when Eq. 13 holds, since in that case the test particle effective temperature $T_{\text {eff }}$ defined via the relationship:

$$
T_{\text {eff }} \exp \left(-E / K_{B} T_{\text {eff }}\right)=\left[T_{1} \exp \left(-E / K_{B} T_{1}\right)+T_{2} \exp \left(-E / K_{B} T_{2}\right)\right] / 2
$$

becomes simply $T_{\text {eff }} \simeq\left(T_{1}+T_{2}\right) / 2$ up to terms of order $\left(E / K_{B} T_{1}\right)^{2},\left(E / K_{B} T_{2}\right)^{2}$.

If we examine the lower thermalization temperature of the test particle more carefully, it seems to be a direct result from the shift of the thermalization region. Since this shifted thermalization region is not the optimal region for single bath thermalization, the test particle does not thermalize with each of the two baths effectively. If we focus on the new optimal thermalization region, we can see that, although the test particle does not reach the average temperature of the two baths, it does get close to the average of two temperatures when the test particle thermalizes with the two baths separately in a narrow frequency range as shown in Fig. 10.

Both these features are also present in the complementary situation of two heat baths at the same temperature but with non overlapping density of states, as shown in Fig. 11. In this case, while the test particle thermalizes at the common temperature of the heat baths when they are acting independently, in the intermittent case the two thermalization regions are redshifted and the test particle's temperature is nearly half of the heat baths'. One possible explanation for this half thermalized temperature is that the test particle cannot absorb much energy from the bath when its frequency is outside the frequency band of the bath as it has been shown in the one bath case. Therefore a low frequency test particle does not receive energy from the particles in the high frequency bath, and thus it is as if the test particle is interacting with one bath at temperature 10 and the other bath at temperature 0 , and likewise for the high frequency test particle. In other words, what matters is a sort of effective spectral temperature for the test particle, and if there are no oscillators around its proper frequency the corresponding bath is effectively at zero temperature. In Fig. 12 we show the combined effect of heat baths at different temperatures and different density of states. In the left plot, we display the case of the cold bath having oscillators at lower frequency (and the hot bath with density of states shifted towards higher frequencies), with the test particle showing the expected thermalization to each bath at the expected temperature and in the same spectral region. When both baths are acting, the test 
particle displays similar features to the previous figures, i.e. an effective temperature about half of the temperature of the bath operating in the same frequency region, and a redshift of the thermalization spectrum. In the right plot, we present the dual situation, with the cold bath having oscillators at higher frequency.

\section{CONCLUSIONS}

We have studied finite resource reservoirs, showing that thermalization of a test particle occurs only under specific conditions in both the case of continuous interaction with a single bath and intermittent interaction with two baths with different features. These results may have an impact on the investigations of equilibrium and nonequilibrium quantum statistical mechanics of nanostructures, as first discussed in [36] in the framework of quantum-limited electromechanical tunneling transducers. Since then, many groups have pushed the sensitivity of displacement transducers for micro and nanoresonators near the quantum limit [37]. Three areas of current research could benefit from our discussions. In [6], a phenomenon of cooling has been interpreted as due to quantum back-action of the read-out system. A more economical interpretation, without necessarily invoking the quantum features of the measurement apparatus, is instead available by imagining the effective temperature of the nanoresonator as resulting from the competition between two effective heat baths at different temperatures. This is also in line with the phenomenon of cold damping introduced eight decades ago [3], and demonstrated for macroscopic resonators in [7]. From our perspective, the study of the thermalization of a particle in simultaneous interaction with two heat baths should shed light on this phenomenon already at the classical level, especially focusing on the energy distribution which, in the presence of finite resources, is not necessarily of Boltzmann nature, and it could help to understand some intriguing features of the effective temperature found in the demonstration reported in [6]. A second class of experiments which may benefit from our discussion are the ones involving very high frequency nanoresonators, as pioneered in [38]. In our framework the existence of discrete jumps could be attributed to non-equilibrium features of the test particle, in this case a high-frequency mode of a nanomechanical structure. Due to its geometry and size, the nanoresonator may have a density of states of the phonon bath with Debye frequency much less than in the corresponding bulk situation. This should be confirmed by both dedicated experiments on thermal properties, such as specific heat and heat conductivity, and $a b$ initio calculations of the density of states. Our conjecture is that for lower dimensionality structures the Debye frequency and the larger amount of relevant states may lie below the GHz range, i.e. below the resonator frequency. The effective temperature felt by the test particle could then be quite different from the one expected by measuring the external bath to which the nanoresonator is coupled. Non-equilibrium distributions are also expected in the case, not considered here, of test particles schematized in the quantum realm, for which even with a proper choice of observables deviations from the Boltzmann energy distribution should be observed [39]. Finally, we believe that the careful design of some environments could help to extend quantum nondemolition measurements [40, 41] in nanomechanical devices. It is well known that quantum nondemolition measurements are suitable only for the case of harmonic oscillators. Their extension to free particles in the form of measurements on carefully prepared contractive states [42, 43] or Schroedinger cat states [44] is quite problematic in practice. Furthermore, the extension of quantum nondemolition measurements to generic nonlinear systems is also difficult, as it requires a specific knowledge of the initial state of the system [45]. We believe that proper engineering of the environment surrounding the nanoresonator, with blockade of some frequency range for the exchanged phonons [46 49], corresponding in our model to a proper preparation of the density of states of the bath, will provide a robust and general way to reach and surpass the standard quantum limit.

\section{Acknowledgments}

S.T.S. acknowledges partial support from the Mellam Foundation at Dartmouth.

[1] A. Einstein, Ann. Phys. 19, 289 (1906).

[2] A. Einstein, Ann. Phys. 19, 371 (1906).

[3] L.S. Ornstein, H.C. Burger, J. Taylor, and W. Clarkson, Proc. Roy. Soc. 115, 391 (1927).

[4] A.O. Caldeira and A.J. Leggett, Phys. Rev. Lett. 46, 211 (1981).

[5] A.O. Caldeira and A.J. Leggett, Ann. Phys. 149, 374 (1983).

[6] A. Naik, O. Buu, M.D. LaHaye, A.D. Armour, A.A. Clerk, M.P. Blencowe, and K.C. Schwab, Nature 443, 193 (2006).

[7] H. Hirakawa, S. Hiramatsu, and Y. Ogawa, Phys. Lett. A 63, 199 (1977).

[8] S.T. Smith and R. Onofrio, Eur. Phys. J. B 61, 271 (2008).

[9] C. Kipnis, C. Marchioro, and E. Presutti, J. Stat. Phys. 27, 65 (1982). 
[10] P. Hänggi, Generalized Langevin Equations: A Useful Tool for the Perplexed Modeler of Nonequilibrium Fluctuations? in: Stochastic Dynamics, L. Schimansky Geier and T. Pöschel, eds., Lecture Notes in Physics, vol. 484 15-22 (Springer Verlag, Berlin, 1997).

[11] H. Kohler and F. Sols, Phys. Rev. B 72, 180404(R) (2005).

[12] S. Trimper, Phys. Rev. E 74, 051121 (2006).

[13] H. Kohler and F. Sols, New J. Phys. 8, 149 (2006).

[14] P. Visco, J. Stat. Mech.: Theory and Experiment (2006) P06006.

[15] L. Bertini, A. Del Sole, D. Gabrielli, G. Jona-Lasinio, and C. Ladim, J. Stat. Mech.: Theory and Experiment (2007) P07014.

[16] G.W. Ford, M. Kac, and P. Mazur, J. Math. Phys. 6, 504 (1965).

[17] G.W. Ford and M. Kac, J. Stat. Phys. 46, 803 (1987).

[18] V. Hakim and V. Ambegaokar, Phys. Rev. A 32, 423 (1985).

[19] M. Patriarca, Nuovo Cimento B 111, 61 (1996).

[20] B.L. Hu, J.P. Paz, and Y. Zhang, Phys. Rev. D 47, 1576 (1993).

[21] H. Mori, Prog. Theor. Phys. 33, 423 (1965).

[22] R. Kubo, Rep. Prog. Phys. 29, 255 (1966).

[23] C. Presilla, R. Onofrio, and M. Patriarca, J. Phys. A: Math. Gen. 30, 7385 (1997).

[24] A. Carcaterra, Mech. Syst. and Signal Proc. 16, 905 (2002).

[25] A. Carcaterra, J. Sound and Vibration 288, 751 (2005).

[26] A. Carcaterra and A. Akay, J. Acoust. Soc. Am. 115, 683 (2004).

[27] C.E. Celik and A. Akay, J. Acoust. Soc. Am. 108, 184 (2000).

[28] A. R. Plastino and A. Plastino, Phys. Lett. A 193, 140 (1994).

[29] A.B. Adib, M.P. Almeida, J.S. Andrade, A.A. Moreira, Physica A 322, 276 (2003).

[30] J.S. Andrade, M.P. Almeida, A.A. Moreira, G.A. Farias, Phys. Rev. E 65, 036121 (2002).

[31] F.Q. Potiguar, U.M.S. Costa, Physica A 321, 482 (2003).

[32] H.B. Prosper, Am. J. Phys. 61, 54 (1993).

[33] F.Q. Potiguar and U.M.S. Costa, Physica A 344, 614 (2004).

[34] A.V. Plyukhin, Europhys. Lett. 75, 15 (2006).

[35] L. Viola and S. Lloyd, Phys. Rev. A 58, 2733 (1998).

[36] C. Presilla, R. Onofrio, and M.F. Bocko, Phys. Rev. B 45, 3735 (1992).

[37] V.B. Braginsky and F.Ya. Khalili, Quantum Measurements (Cambridge University Press, Cambridge, 1992).

[38] A. Gairdarzhy, G. Zolfagharkhani, R.L. Badzey, and P. Mohanty, Phys. Rev. Lett. 94, 030402 (2005).

[39] R. Onofrio, Europhys. Lett. 11, 695 (1990).

[40] C.M. Caves, K.S. Thorne, R.W. Drever, V.D. Sandberg, and M. Zimmermann, Rev. Mod. Phys. 52, 341 (1980).

[41] M.F. Bocko and R. Onofrio, Rev. Mod. Phys. 68, 755 (1996).

[42] H.P. Yuen, Phys. Rev. Lett. 51, 719 (1983).

[43] M. Ozawa, Phys. Rev. Lett. 60, 385 (1988).

[44] L. Viola and R. Onofrio, New J. Phys. 5, 5 (2003).

[45] M.B. Mensky, R. Onofrio, and C. Presilla, Phys. Rev. Lett. 70, 2825 (1993).

[46] K.R. Patton and M.R. Geller, Phys. Rev. B 67, 155418 (2003).

[47] M.R. Geller, Phys. Rev. B 70, 205421 (2004).

[48] S.-X. Qu, A.N. Cleland, and M.R. Geller, Phys. Rev. B 72, 224301 (2005).

[49] I. Wilson-Rae, Phys. Rev. B 77, 245418 (2008). 